\title{
RECHTSTEXTE IM VERGLEICH. DAS BEISPIEL VON DEUTSCHEN UND ITALIENISCHEN UNIVERSITÄREN LEHRBÜCHERN
}

\section{Einleitung}

Die kontrastive Fachsprachenforschung hat sich seit einigen Jahren die Aufgabe gestellt, Gemeinsamkeiten und Unterschiede zwischen Fachtexten/Fachtextsorten unterschiedlicher Fächer und/oder unterschiedlicher Sprachen zu zeigen. Dadurch soll einerseits die Annahme über die Existenz kultur- und nationalspezifischer Denktraditionen bewiesen werden, die fachsprachliches Handeln prägen und steuern; ${ }^{1}$ andererseits sollen durch den interlingualen Vergleich für dieselben Textsorten konkrete Hinweise für Bereiche wie Übersetzungswissenschaft und Fremdsprachendidaktik - wo der kontrastive Ansatz immer mehr an Bedeutung gewinnt - gegeben werden.

Die Untersuchung zu deutschen und italienischen Rechtstexten, die hier vorgestellt wird, ist innerhalb des Projekts zur kontrastiven Rechtstextlinguistik entstanden, das im Fachbereich "Sprache und Recht" an der Europäischen Akademie Bozen angesiedelt ist. Bei diesem Projekt geht es darum, Ähnlichkeiten und Besonderheiten von Rechtstexten unterschiedlicher Art - normative, wissenschaftliche, didaktische Texte - am Sprachenpaar Deutsch-Italienisch hervorzuheben, um einen sowohl theoretischen als auch praktischen Beitrag zur Fachsprachenforschung zu leisten. Das Projekt begann mit der Analyse von normativen Texten: so wurden deutsche und italienische Gerichtsurteile im Hinblick auf die Makrostruktur analysiert, während der Vergleich von Gesetzesstexten auf die sprachliche Realisierung von typischen gesetzlichen Inhalten wie Rechten, Pflichten, Verboten und Erlaubnissen fokussierte. ${ }^{2}$ Nun wird die Aufmerksamkeit auf weitere juristische Textsorten gelenkt, die eher der wissenschaftlichen bzw. didaktischen Kommunikation dienen.

Im vorliegenden Beitrag werden die ersten Ergebnisse der kontrastiven Analyse von universitären Lehrbüchern vorgestellt, die Studenten der Rechtswissenschaft oder der Politikwissenschaft in das Europarecht einführen sollen. Ziel einer solchen

1 Vgl. dazu Fiedler (1994: 68), Busch-Lauer (1994: 71), Gläser (1992: 78ff.), Gnutzmann (1992: 268), Clyne (1993). Für eine allgemeine Einführung zur Thematik siehe Pöckl (1995).

2 Vgl. Arntz (1995: 137-162) und Veronesi (1997). 
Untersuchung ist es, Strategien und Mittel hervorzuheben, die der Autor zur Didaktisierung der Inhalte verwendet; damit soll ein Bild über den Dialog gewonnen werden, der zwischen einem Rechtswissenschaftler (als Fachmann) und einem Studenten (als Laien in der Ausbildung) innerhalb zweier verschiedener juristischer, didaktischer und kultureller Traditionen entsteht.

\section{Zur Definition des Untersuchungsgegenstandes}

\subsection{Die Textsorte "universitäres Lehrbuch" und die kommunikative Situation}

Von einem typologischen Gesichtspunkt aus können Lehrbücher als Textsortenklasse betrachtet werden, die verschiedene textuelle Realisierungen hat (Schullehrbücher, universitäre Lehrbücher) (vgl. Göpferich 1995). Solche Realisierungen sind weiter je nach Fachsprache und Ausbildungsniveau zu differenzieren, da sich die Didaktisierung der Inhalte vermutlich je nach Fachgebiet und je nach angenommenem Wissensniveau der Adressaten anders vollzieht.

Weiterhin ist das Lehrbuch auch innerhalb der jeweiligen Fachtexttypologie zu sehen; für das Rechtsgebiet gehört das Lehrbuch zusammen mit Monographien, Fachzeitschriftenartikeln, Kommentaren und Vorarbeiten für die Gesetzgebung den Textsorten an, die für die Rechtslehre typisch sind. ${ }^{3}$ Bei solchen Textsorten sind Lehrbücher expositorische Texte, die (ähnlich wie Enzyklopädien) eine erklärende Funktion haben. Sie erfüllen die Aufgabe, im Rahmen der Ausbildung jemandem einen neuen Sachverhalt darzustellen und deutlich zu machen. Im Vergleich mit anderen juristischen Textsorten wie normativen Texten, wo Textstruktur und sprachliche Mittel stark konventionalisiert sind und wo daher der Autor formalen und sprachlichen Zwängen unterliegt, sind Lehrbücher für den Autor nicht so verbindlich, ${ }^{4}$ so daß hier eine größere Auswahl an Strategien und Mitteln getroffen werden kann, deren Analyse daher auch im Hinblick auf eventuelle nationalspezifische Denk- und Argumentationsstile nutzbar sein kann.

Was den konkreten Kommunikationskontext betrifft, in dem die hier analysierten Lehrbücher zum Europarecht verwendet werden, so muß man sich vor Augen halten, daß Europarecht sowohl in Deutschland als auch in Italien ziemlich am Ende des

3 Siehe dazu Cavagnoli / Veronesi (1996).

4 Sabatini (1990: 694-700) schlägt für die Textklassifikation eine Makrotypologie vor, die aufgrund des pragmatisch orientierten Parameters "Grad der Verbindlichkeit, die der Autor von der Interpretation des Lesers verlangt" ("grado di rigidità del vincolo che l'autore pone all'interpretazione del lettore") drei Grundkategorien enthält: Texte mit sehr verbindlichem Diskurs, mit mittelmäßig verbindlichem Diskurs und mit wenig verbindlichem Diskurs. Lehrbücher gehören hierbei zusammen mit Traktaten, Essays und Enzyklopädien zu den expositorischen Texten, welche wie die informativen Texten (Sachtexte, Pressetexte u.ä.) durch eine mittelmäßige Verbindlichkeit des Diskurses gekennzeichnet sind. 
Studiums behandelt wird: es ist ein komplexes Gebiet, bei dem viele juristische Kenntnisse vorausgesetzt werden. Solche Lehrbücher sind als Begleithilfe zu Vorlesungen oder Seminaren gedacht und werden vom Studenten autonom verwendet.

\subsection{Die Korpusanalyse}

Das analysierte Korpus wurde fünf italienischen und fünf deutschen universitären Lehrbüchern zum Europarecht entnommen; dabei wurden die Kapitel bzw. die Kapitelabschnitte untersucht, die dasselbe Thema behandeln, nämlich das Verhältnis vom EG-Recht zum nationalen Recht und die daraus folgende Problematik der Anwendbarkeit/Anwendung des EG-Rechts in den Mitgliedstaaten, was auch zu den am meisten diskutierten Fragen dieses Rechtsgebiets gehört. ${ }^{5}$

Bei der Analyse wurde versucht, einerseits von außen nach innen und anderseits vom Ganzen zum Einzelnen zu gehen, um solche Texte in ihrer Komplexität einigermaßen fassen zu können. Die Lehrbücher wurden deshalb zunächst als textuelle Objekte betrachtet, wie sie sich einem Leser darbieten:

a) mit einem Vorwort, das sie vorstellen und begründen soll,

b) mit einer gewissen Gliederung und einer gewissen graphischen Gestaltung, an welcher sich der Leser orientieren soll,

c) mit zusätzlichen Hilfen, die die Arbeit am Text erleichern sollen.

In einer zweiten Phase wurde auf die sprachliche Ebene eingegangen, um herauszufinden, wie sich der Dialog zwischen Autor als Fachmann und Leser als Laien, der aber am Ende seiner Ausbildung steht, im Text selbst konkret realisiert. So wurden explizite Signale des Autors gesucht, die die Informationsvermittlung erleichtern und den Leser in der Textrezeption unterstützen können:

a) deiktische Ausdrücke, die die Orientierung des Lesers im textuellen Raum steuern;

b) Erklärungen und Präzisierungen, die komplexe Sachverhalte deutlich machen sollen;

c) Explizierung von kausalen Verhältnissen, die die argumentativen Schritte des Autors für den Leser hervorheben.

Solche Parameter erschienen im Hinblick auf die Didaktisierung von komplexen rechtlichen Inhalten, wie es bei Europarecht-Lehrbüchern vorkommt, besonders aussagekräftig; allerdings kann die vorliegende Analyse aufgrund der Begrenztheit sowohl der Vergleichsgrößen ${ }^{6}$ als auch des analysierten Korpus ausschließlich als

5 Insgesamt umfaBt das Korpus 108 'effektive' Seiten (d.h. ohne Zitate) für das Italienische und 72 Seiten für das Deutsche, wobei sich die Seitenzahl in den beiden Sprachen durch das Vorhandensein von kleingedruckten Textabschnitten in allen deutschen Texten ungefähr gleicht. Im Folgenden wird auf die Lehrbücher durch die Autorennennung Bezug genommen (Beutler/Bieber; Bleckmann; Oppermann; Schweitzer/Hummer; Streinz; Ballarino; Ferrari Bravo; Lauria; Pocar; Zanghi); genaue Angaben zum Korpus im Quellenverzeichnis. 
explorativ betrachtet werden, in der Hoffnung allenfalls, Tendenzen der Textualisierung in den beiden Sprachen aufzuzeigen, die weiter zu überprüfen sind. Ziel dieser Analyse war also in erster Linie, eine Basis für gründlichere Untersuchungen an solchen Texten zu schaffen und die Anwendbarkeit der ausgewählten Vergleichskriterien für die Textsorte "universitäres Lehrbuch" zu erproben.

\section{Der Ansatz von außen}

\subsection{Das Vorwort, oder wie der Autor den Leser sieht}

Bei der Analyse der Vorwörter ging es darum, ob bzw. inwieweit der Autor seine Leserschaft direkt anspricht und wie er diesen Dialog durchführt. Das Vorwort wird in diesem Zusammenhang als 'Eingang' zum eigentlichen Text, also als textueller Ort ${ }^{7}$ gesehen, wo der erste Kontakt zwischen Autor und Leser stattfindet, und vor allem, wo der Autor sich explizit in seiner Rolle als Textproduzent - d.h. mit bestimmten Intentionen und Zwecken, mit bestimmten Methoden und mit einer bestimmten Leserschaft im Auge - vorstellt. Es ist der erste 'offizielle', institutionalisierte Moment, wo sich die Beziehung Autor-Leser konstituiert. ${ }^{8}$

Bei allen Vorwörtern kommen bestimmte Handlungen - wenn auch in unterschiedlicher Gewichtung - vor, die zur textuellen Charakterisierung zu gehören scheinen: Der Autor erklärt, wie das Werk entstanden ist; ggfs. nimmt er Bezug auf vorherige Auflagen; er äußert seine Meinung über die Besonderheiten des dargestellten Stoffes (hier die zunehmende Bedeutung und die letzten Veränderungen im Europarecht); er dankt Mitarbeitern, Kollegen, Familie, Sachbearbeitern und Verlag

$6 \mathrm{Zu}$ den verschiedenen Analyseansätzen in der kontrastiven Fachsprachenforschung und zur Problematik der Auswahl des tertium bzw. der tertia comparationis siehe insbesondere Oldenburg (1995).

7 Genauer gesagt zählt das Vorwort zu den internen paratextuellen Elementen (Peritext) (vgl. Genette 1987), die zusammen mit den externen paratextuellen Elementen (Epitext) die Aufgabe haben, die Lektüre zu steuern. Streng genommen, stellen Vorwörter nicht unbedingt den ersten Kontakt zwischen Autor und Leser (der schon durch Elemente wie Titel, Autorname usw. entsteht) dar; im Zusammenhang dieser Untersuchung erscheint jedoch eine solche Präzisierung nicht besonders relevant. Über das Vorwort als Textsorte siehe I. Thonhauser-Jursnick, "«Mein besonderer Dank gilt» - Das Vorwort. Strukturen wissenschaftlicher Dankbarkeit" in diesem Band.

8 Das bedeutet natürlich nicht unbedingt, $\mathrm{da} B$ Vorwörter auch tatsächlich von den 'institutionellen' Adressaten - den Studenten - gelesen werden; Vorwörter können auch gleichzeitig als Kommunikationsmittel innerhalb einer wissenschaftlichen Gemeinschaft dienen, was das Vorhandensein von Handlungen wie 'Danken', 'Loben', 'die persönliche Meinung über gewisse Themen äußern', 'von sich selbst erzählen' - die alle in Vorwörtern vorkommen können -, zu begründen scheint. Dadurch verliert aber das Vorwort seinen 'offiziellen' Charakter als Einleitung für den primären Leser (hier Studenten) nicht, so daß dessen Analyse interessante Hinweise auf die Beziehung Autor - Leser geben kann. 
(was in größerem Maße bei den deutschen Texten gemacht wird, wo z.T. auch Rechtsreferendaren und Lektoren gedankt wird).

Hinzu kommen Inhalte, die eher dem persönlichen Stil des Autors zuzuschreiben sind: Ein italienischer Autor (Ferrari Bravo) äußert z.B. scharfe Kritik an den Veränderungen, die in der Studienordnung eingeführt wurden; ein deutscher Autor (Bleckmann) erklärt sich bereit, Hinweisen und Verbesserungsvorschlägen "an die unten genannte Adresse" entgegenzukommen, nachdem er seine Bemühungen erwähnt hat, die "z.T. zu Recht kritisierten" formalen Mängel der vorherigen Auflage zu beseitigen (für "Hinweise, Anregungen und Kritik" sind auch Schweitzer/Hummer "jederzeit" dankbar); der Autor öffnet dem Leser seine/ihre persönliche Sphäre, indem er/sie, wie die italienische Felicetta Lauria, über den vorbildlichen Vater spricht oder indem er, wie Oppermann, die behandelte Disziplin als "einen der liebsten Forschungsgegenstände" bezeichnet, seine Erfahrung in der Europa-Abteilung des Bonner Bundesministeriums als "eine mit vielen guten Erinnerungen verknüpfte Zeit" erwähnt und hofft, daß ihn das Gespräch mit seiner Frau vor "zu viel juristischem Rigorismus" bewahrt habe.

Neben solchen obligatorischen und fakultativen Teilen ist aber auch ein Bezug zur Leserschaft immer vorhanden, sei es als explizite Erwähnung der Adressaten, sei es als Darstellung des zugrundeliegenden didaktischen Konzepts, sei es als Begründung für die vom Autor vollzogene Themenauswahl.

In dieser Hinsicht scheint sich eine erste Differenzierung zwischen deutschen und italienischen Vorwörtern abzuzeichnen: Obwohl der Bezug zur intendierten Leserschaft in beiden Sprachen vorhanden ist, ist bei den deutschen Autoren - insgesamt gesehen eine etwas größere Aufmerksamkeit bzw. eine höhere Thematisierung des Kommunikationspartners festzustellen.

Bei drei deutschen Texten (Bleckmann, Schweitzer/Hummer, Streinz) wird nämlich schon im ersten Abschnitt des Vorworts auf die "Ausbildungs- und Prüfungsordnungen für Juristen", auf "Studenten der Rechts-, Sozial- und Politikwissenschaften" und auf die "juristische und wirtschaftswissenschaftliche Ausbildung und Praxis" Bezug genommen, was auch als Begründung für die getroffene Themenauswahl benutzt wird.

In den drei Vorwörtern werden dann weiter im Text das didaktische Konzept und die angebotenen Hilfsmittel erwähnt (so z.B. die Einbindung von "Fällen mit Lösungen" in der Darstellung bei Streinz, oder die Ausstattung des Textes mit Randnummern in Bleckmann, der auch ein "ausführliches" Sachverzeichnis, ein Rechtsprechungsregister und ein Artikelregister zum "raschen Zugriff auf die Problemfelder" zur Verfügung stellt). Bei Schweitzer/Hummer nimmt die Darstellung des didaktischen Konzepts - in Form einer Auflistung - den größten Teil des Vorworts ein, wo auch auf weitere Lernmaterialien (Textbuch, Übungsbuch, Fälle-Buch) verwiesen wird, die die Autoren selbst als Begleitwerk zum Lehrbuch verfaßt haben.

Bei den zwei übrigen Texten (Oppermann, Beutler/Bieber) stellt man hingegen eine gewisse Polarisierung fest: Während das Vorwort Oppermanns sehr persönlich und 'autorenzentriert' wirkt, was immerhin den Autor nicht daran verhindert, auch 
Studenten zu danken, wird der Adressat "Studenten" im Vorwort des gemeinsamen Werkes von den vier Autoren Beutler/Bieber/Pipkorn/Streil gar nicht thematisiert, selbst da nicht, wo es um das "Ziel" der vierten Auflage geht.

Bei den italienischen Vorwörtern ist das Bild etwas anders: Nur bei einem Text (Pocar) werden im ersten Abschnitt Adressaten und Ziele des Buches erwähnt, während bei den übrigen vier Texten zunächst über Probleme und Entwicklungen des Europarechts gesprochen wird, um erst nach mehreren Absätzen zur Didaktik überzugehen.

Alle italienischen Autoren zeigen weiterhin die Neigung, eine persönliche Perspektive als 'Autoren' anzunehmen, bevor von Didaktik und Studenten die Rede ist: die Thematisierung der Adressaten entsteht nämlich durch die vorherige Thematisierung des Buches als persönliches Produkt, als Ergebnis von universitären Vorlesungen oder als Ergebnis einer akademischen bzw. beruflichen Erfahrung. So bei Pocar

La presente trattazione, che trova la sua origine nelle lezioni di diritto delle Comunità europee da me tenute diversi anni orsono presso la Facoltà di scienze politiche dell'Università degli studi di Milano, risponde in via primaria all'esigenza di offrire agli studenti, agli operatori del diritto e a quanti si accostino al fenomeno comunitario uno strumento aggiornato [...].

\section{bei Ballarino}

Rileggendo il testo del mio manuale, con tutte le varianti e le modificazioni introdotte nel corso delle quattro edizioni che ha avuto, credo di poter presentare le seguenti riflessioni. a) [...]. b) [...]. c) Nella didattica della disciplina, destinata a studenti universitari, occorre tenere conto dei termini di riferimento che ha lo studente. Questo proposito mi ha indotto a...[...].

\section{bei Zanghi}

Oltre dieci anni di esperienza didattica presso la Scuola Superiore della Pubblica Amministrazione [...] mi hanno indotto ad un approccio pragmatico della materia [...]. Il continuo confronto con i funzionari dell'Amministrazione centrale e regionale mi ha consentito di affinare nel tempo le conseguenti metodologie didattiche. Rientrando adesso nell'insegnamento universitario d'origine, ho ritenuto opportuno porre a profitto del diverso pubblico l'accennata esperienza. A ciò mi ha spinto la consapevolezza che la formazione universitaria, specie in una Facoltà come quella di Scienze Politiche [...].

bei Lauria, ${ }^{9}$ wo die Autorin im Vorwort zur zweiten Auflage schreibt:

Il rapido esaurirsi della prima edizione di questo Manuale, destinato in origine ai miei studenti dell'Università di Milano ed agli allievi ufficiali dell'Accademia della Guardia di Finanza di Bergamo, è prova del profondo interesse [...].

9 Hier wird die Einleitung von einem anderen Autor verfaßt, der erst am Ende seiner Präsentation die Entstehung des Buches erwähnt: "[...] è certo che il lavoro della prof. Lauria, nato dai corsi di 
und Ferrari Bravo:

Il rapido esaurimento delle mie Lezioni di diritto delle Comunità Europee, apparse nel 1992, mi ha creato una serie di problemi.

Die Themenauswahl wird in drei Lehrbüchern direkt auf die besondere Leserschaft bezogen, während nur in zwei Lehrbüchern (Pocar, Zanghì) auch die Hilfsmittel (detaillierte Bibliographie und Verzeichnisse der Rechtsprechung und der zitierten Gesetzgebung in Pocar, ähnliche Verzeichnisse in Zanghi) erwähnt werden. In den zwei übrigen Lehrbüchern wird über den "Leser" in einer ziemlich allgemeinen und konventionellen Weise gesprochen (der Leser werde schließlich über die Wirksamkeit des Werkes urteilen, so heißt in Ferrari Bravo), bzw. er wird gar nicht erwähnt.

Was weiterhin die Darstellungshaltung des Autors gegenüber dem Gegenstand und dem Adressaten betrifft, die durch die Verwendung von Personalpronomina der ersten Person Singular und Plural oder aber durch die Verwendung von unpersönlichen Formen wie dem Passiv signalisiert wird, ${ }^{10}$ so wirken die italienischen Texte persönlicher, da vier von fünf Gebrauch von der ersten Person Singular machen, während bei den deutschen Texten eher die Neigung zur distanzierten Darstellungsweise (mit vorwiegenden Passiv-Konstruktionen) festzustellen ist. ${ }^{11}$

\subsection{Das Lehrbuch als Text: Gliederungsebenen, Hilfsmittel, nichtsprachliche Textteile, typographische Signale}

Nach der Analyse des Vorworts als ersten textuellen Ortes der Kommunikation zwischen Autor und Leser ist es nun möglich, einen Schritt in den Text "Lehrbuch" zu machen und nachzuprüfen, unter welchen Bedingungen deren Dialog stattfinden kann, d.h. mit welcher Textstruktur und mit welchen Hilfsmitteln sich der Leser im Text zurechtfinden muß.

Es geht hier um formale (paratextuelle) Merkmale des Textes, die zwar noch nicht zur Textpartitur gehören, die aber eine Steuerungsfunktion in der Textrezeption ausüben können, indem sie den Text nach einem gewissen Schema organisieren, thematische Einheiten signalisieren und die 'rohen' sprachlichen Zeichen mit zusätzlichen Vertiefungs- und Orientierungsmitteln ergänzen. Es wurde deshalb als erster Schritt in den Text die Textstruktur untersucht: die Art und die Tiefe der Gliederung des K. itels, dessen interne Struktur, die Verwendung von nichtsprach-

diritto comııı1tario tenuti da ormai cinque anni nella Facoltà di Scienze Politiche dell'Università di Milano costituisce uno strumento essenziale [...]").

10 Vgl. Gläser (1994: 75-78).

11 Wenn die italienischen Autoren durch die Verwendung von Personalpronomina nicht so distanziert wie die deutschen wirken, so ist dabei auch $z u$ berücksichtigen, daß eine solche 'Personalisierung' auch dadurch entsteht, daß die ersteren einem persönlicheren Duktus haben. 
lichen Elementen (Schaubildern, Tabellen) und das Vorhandensein zusätzlicher Nachschlagmittel (Register, Literaturhinweise). ${ }^{12}$

Was die Gliederung der einzelnen Kapitel angeht, so zeichnet sich bei dem Vergleich eine größere Detaillierung bei den deutschen Texten ab: Während nämlich im italienischen Korpus zwei Lehrbücher drei Gliederungsebenen aufweisen, weitere zwei nur eine Ebene (bei einem Lehrbuch - Ballarino - ist diese auch nicht formal alphabetisch und/oder numerisch markiert) und ein Lehrbuch (Ferrari Bravo) sogar keine interne Gliederung aufweist (was auch mit seinem Status als "Vorlesungsbuch" zusammenhängt), sind drei deutsche Texte in drei Ebenen gegliedert und die übrigen Texte (Streinz, Bleckmann) weisen jeweils vier und fünf auf. Bei den deutschen Autoren ist somit die Tendenz zu erkennen, Themen in Unterthemen zu gliedern und diese weiter zu zerlegen, so da $ß$ jedes Thema bzw. Unterthema sozusagen getrennt und graphisch isoliert behandelt wird, was auch die inhaltliche Hierarchie der Themen hervorhebt. Bei den italienischen Autoren hingegen werden nicht so viele 'Unterteilungen' gemacht: Unterthemen werden innerhalb eines und desselben Kapitelteils in einer einheitlichen Behandlung präsentiert.

Bei der internen Struktur des Kapitels werden Unterschiede in der Stoffdarbietung noch deutlicher: In allen deutschen Texten kommen neben einem Haupttext auch kleingedruckte Textabschnitte vor, die in den meisten Texten (vier von fünf) nicht nur zum Zitieren von Urteilen oder Gesetzestexten, sondern auch zur Vertiefung der einzelnen Themen dienen. Hingegen kommen solche graphisch isolierbaren Texteinheiten nur in zwei italienischen Texten vor, wobei sie in einem Text nur für Zitate verwendet werden. Bei den italienischen Texten (vier von fünf) ist auch das Fehlen von Fußnoten zu bemerken, was sonst typisch für expositorische und wissenschaftliche juristische Texte ist; dieser Verzicht ist im Deutschen weniger ausgeprägt (Fußnoten werden bei drei Texten verwendet).

Was weiterhin besondere didaktische Hilfen innerhalb des Kapitels betrifft, so enthalten alle Texte in den beiden Sprachen (außer einem italienischen Text, der allerdings dieselbe Information in Fußnoten vermittelt) weitere bibliographische Hinweise, sei es nur am Ende des Kapitels oder auch zu den einzelnen Abschnitten (in zwei ital. und in vier deutschen Lehrbüchern).

Was aber noch mehr auffällt und ein wichtigeres Zeichen für 'Leserfreundlichkeit' ist, ist die didaktische Verwendung, bei zwei deutschen Texten, von Fragen am Ende eines Themas, von Fällen, die vom Studenten selbst gelöst werden können (mit Lösungen am Ende des Kapitels) und von (graphisch hervorgehobenen) Beispielen zu den erläuterten Prinzipien, die im italienischen Korpus gänzlich fehlen. Zusätzliche

12 Einige der hier genannten Elemente könnten auch als "advance organisers" gesehen werden. Diese beschreibt Gläser (1995: 87) folgendermaßen: "Metacommunicative disposition signals (advance organisers) [...] alleviate the reader's orientation in the progression of the text and the reception of the information conveyed. These may be sub-headings, the enumeration of items by means of numbers or letters, different types of print, ect." 
Orientierungshilfen bekommt der Leser bei zwei italienischen Lehrbiichern durch ein Inhaltsverzeichnis zum jeweiligen Kapitel (das betrifft gerade die zwei Texte, die drei Gliederungsebenen haben), während bei drei deutschen Texten die Abschnitte mit Randnummern versehen sind.

Schaut man dann auf andere zusätzliche Hilfen, die zum Instrumentarium eines Lehrbuchs bzw. eines wissenschaftlichen Buches gehören, wie z.B. Literaturangaben, Sachregister $\mathbf{u}$. ähnliches, stellt man fest, daß bei allen Lehrbüchern ein sozusagen 'minimaler harter Kern' vorhanden ist, der aus Inhaltsverzeichnis und Literaturhinweisen besteht, um den sich weitere zusätzlichen Hilfen für den Leser gruppieren und die auf eine größere Aufmerksamkeit für den deutschen Leser zu deuten scheinen: so ist z.B. in zwei deutschen Texten vor dem eigentlichen Inhaltsverzeichnis auch eine Inhaltsübersicht zu finden, was in keinem italienischen Text vorkommt; alle deutschen Texte sind außerdem mit einem Sachregister und mit einem Rechtsprechungsverzeichnis versehen, welche nur in zwei italienischen Büchern zu finden sind.

Was nichtsprachliche Textteile wie Bilder und Tabellen angeht, werden solche Mittel, deren didaktische Wirkung weitgehend anerkannt ist ${ }^{13}$ und deren Verwendung also gerade für die Textsorte "Lehrbuch" zu erwarten wäre, in sehr begrenzten Maße angewendet.Allerdings ist dabei bemerkenswert, daß die wenigen Schaubilder und Tabellen gerade in (drei) deutschen Texten vorkommen. Einerseits hängt das mit dem besonderen Charakter der Rechtstexte im allgemeinen zusammen, wo (außer eventuell in Enzyklopädien) von Bildern kaum Gebrauch gemacht wird; andererseits kann das auch mit dem höheren Fachlichkeitsgrad von Europarecht-Lehrbüchern zusammenhängen, die sich aufgrund ihrer späteren Benutzung im Studium von anderen (juristischen) Lehrbüchern zu unterscheiden scheinen.

Gliederung, Kapitelstruktur, nichtsprachliche Mittel - aus diesem ersten Vergleich von Strukturmerkmalen ergibt sich für die beiden Sprachen das folgende provisorische Bild: Einerseits sind die deutschen Texte auf den ersten Blick 'didaktischer', indem sie dem Leser mehrere Hilfen zur Verfügung stellen und indem sie direkt an seine Kooperation durch Fragen, Beispiele und Fälle, die gelöst werden sollen, appellieren; andererseits scheinen sie 'leserorientierter' als die italienischen Texte zu sein, indem sie einen Lesertyp implizieren, dessen Orientierung im Text durch eine starke graphische Signalisierung der einzelnen Textteile (durch die vielen Gliederungsebenen, durch die Alternanz Haupttext/Vertiefungstext, durch mit Randnummern versetzte Absätze) ermöglicht werden soll.

Eine solche Annahme, nach der die deutschen Texte einen stärkeren 'graphischvisuellen' Charakter haben als die italienischen, wird auch von der Analyse der

13 Vgl. dazu Kalverkämper ("Abbildungen [...] bekommen durch die Signale der Fachlichkeit [...] eine instruierende Funktion und haben so eine didaktische Wirkung, die ein fachliches "Ersehen" der Objekte steuert" (1993: 221) und Ylönen ("Tabellen und Abbildungen [...] ermöglichen ein schnelleres Orientieren über den Textinhalt und eine schnellere Rezeption der Informationen des Textes als ein fortlaufender Text ohne nichtsprachliche Teile, und dienen somit zur Sicherung von Verständlichkeit") (1993: 91). 
typographischen Mittel wie Fett, Kursiv, Unterstrichen oder Sperrdruck bestätigt, die zur Hervorhebung von wichtigen Inhalten (Schlüsselwörter, Prinzipien, Verweise) eingesetzt werden können.

Im deutschen Korpus kommen ein Minimum von 7 Hervorhebungen ${ }^{14}$ (Bleckmann) über 20 (Streinz), 22 (Schweitzer/Hummer), 33 (Beutler/Bieber), bis zu einem Maximum von 85 Hervorhebungen (Oppermann) vor, während es in den italienischen Texten jeweils keine Hervorhebung (Pocar), 5 (Ferrari Bravo), 10 (Zanghi), 11 (Lauria) und höchstens 24 (Fett, Kursiv, Sperrdruck, Ballarino) gibt. In all diesen Fällen handelt es sich um die Hervorhebung von Schlüsselwörtern, die das Thema des Abschnitts bzw. des Absatzes zusammenfassen, wie in Streinz (SS. 54-55),

aa) In der Literatur vertretene Auffassungen

Unhaltbar und daher weitgehend aufgegeben wurden folgende Auffassungen:

Lösung anhand der allgemeinen Regeln des Verhältnisses von Völkerrecht und Landesrecht ohne Berücksichtigung [...]

Bundesstaatliche Lösungen, die [...]

Eine verfahrensrechtliche Lösung dahingehend, daß [...]

Pragmatische Lösungen wie eine gemeinschaftskonforme Auslegung [...]

Vertreten wird ein Vorrang des Gemeinschaftsrechts [...]

Die rein europarechtlichen Lösungen gehen [...]

um die Hervorhebung der wichtigsten Elemente im Satz, wie in Beutler (S.95),

[...] Doch wird damit der Vorrang des Gemeinschaftsrechts nicht hinreichend, nämlich nur gegenüber früherem nationalen Recht, nicht aber gegenüber nachfolgendem nationalen Recht gesichert.

oder um die Hervorhebung ganzer Sätze, die einen wichtigen Inhalt (eine Folgerung, ein Prinzip) ausdrücken, wie in Ballarino (S. 241), wo über eine bedeutende Passage in einem Urteil des italienischen Verfassungsgerichtshofs gesprochen wird:

Essa ha tuttavia escluso che il giudice italiano possa autonomamente disapplicare le norme interne successivamente incompatibili con $i$ regolamenti comunitari.

14 Bei der Analyse der typographischen Mittel wurden Hervorhebungen von lateinischen Wörtern und Prinzipien, Fremdwörtern, Titeln von Gesetzen oder Urteilen und Literaturverweisen nicht miteinbezogen, da sie wegen ihres konventionellen Charakters dem Autorziel der inhaltlichen Hervorhebung nicht zugeschrieben werden können. 


\section{Der Ansatz von innen}

\subsection{Der Text als Raum und als Diskurs: textuelle Deixis und Metakommunikation}

Nach der Analyse formeller Elemente der Textstruktur als Orientierungssignale werden nun sprachliche Elemente behandelt, die, wie textuelle Deixis und metakommunikative Äußerungen, eine wichtige Rolle in der Steuerung des Lesers im Text spielen können.

Mit deiktischen Ausdrücken nimmt ein Sprecher auf räumliche und zeitliche Umstände Bezug; sie liefern dabei die "Person-Raum-Zeit Koordinaten" (Lewandowski 1990: 205). Wenn sich die Kommunikation zwischen Sender und Empfänger durch einen Text vollzieht, stellt dieser Text für die Kommunikationspartner also den Raum dar, wo die Interaktion zwischen den beiden stattfindet, und wo die Elemente im Text sich auf eine zeitlich-räumliche Achse strukturieren. Bei diesem textuellen Raum dienen deiktische Ausdrücke dazu, auf vorherige oder nachfolgende 'Zeiten' und 'Räume' im Text zu verweisen und somit eine Verknüpfung zwischen den Elementen herzustellen. Daneben haben sie die Funktion, den Leser zu steuern: sie sind wie Wegweiser für das deiktische Feld und dienen zur globalen Textstrukturierung. ${ }^{15}$

Im Hinblick auf den besonderen Dialog, der zwischen einem Lehrbuchautor und einem Lehrbuchleser entsteht, wurden hier solche deiktischen Mittel isoliert und analysiert, die intratextuelle Verweise zu vorher behandelten Themen herstellen (die somit wieder in Betracht gezogen werden) oder aber die auf später zu behandelnde Themen und Begriffe verweisen (welche daher in der Behandlung antizipiert werden). Weiterhin erschien es interessant zu überprüfen, ob und inwiefern der Autor metakommunikative Äußerungen über seinen eigenen Diskurs macht, die dazu dienen, dem Leser zu signalisieren, was, wie, wann und warum er etwas behandeln wird. Durch solche Angaben macht der Autor die Struktur seiner Behandlung explizit und erleichtert dem Leser die Aufgabe, ihm in seiner Themenentfaltung zu folgen.

Eine solche Explizitierung der inhaltlichen Progression - so wichtig, wie sie auch scheint - ist bei den deutschen Texten nicht vorhanden: nur bei einem Lehrtext (Beutler/Bieber, S. 77) werden am Kapitelanfang Thema und 'Unterthemen' des Kapitels eingeleitet:

Die folgende Darstellung betrifft vor allem die Stellung der Mitgliedstaaten im Rahmen der drei Gründungsverträge. Die [...] Beziehungen der Mitgliedstaaten haben nicht die gleiche Intensität [...]. Sie werden daher im Anschluß und Vergleich abschließend dargestellt (3.4.).

Weiter im Text wird in dem Abschnitt, der die eigentliche Thematik des Rangverhältnisses einleitet, Bezug auf vorher behandelte Themen genommen, um dann

15 Hierzu (Conte 1984: 189), (Gislimberti 1987: 42), Ehlich (1984: 38-40), Vanelli / Renzi (1995: 347-350). 
das neue Thema darzustellen; ähnliches gilt auch für den Abschnitt, der in das Thema der Rechtsprechung der staatlichen Gerichte einleitet, wo am Ende die Themenorganisation der nachherigen Abschnitte erläutert wird (S. 98):

Im folgenden soll daher zunächst ein Überblick über die Entwicklung der Rechtsprechung in den ursprünglichen Mitgliedstaaten und dann in den neu beigetretenen Mitgliedstaaten gegeben werden.

Bei einem weiteren Lehrbuch (Bleckmann) sind auch ähnliche Signale zu finden (vier insgesamt, wobei zwei davon eher einem bibliographischen Hinweis ähneln, da der Autor den "folgende[n] Abschnitt" bzw. den "Text der folgenden Seiten" als überarbeitete Fassungen von vorherigen Veröffentlichungen vorstellt), die allerdings keine genaue 'operative' Information geben:

Wir werden sehen, daß der erste Teil dieser Ausführungen den EuGH und das BVerfG entscheidend beeinflußt hat (S. 744).

Es ist natürlich nicht der Ort, die zahlreichen Schwachstellen der Kelsenschen Theorie umfassend darzulegen. Für unseren Problemkreis kommt es nur auf die Sicht der Existenz und der Kompetenzen der Staaten an (S. 330).

Schließlich finden wir in Oppermann als letzten Satz des Kapitels eine zusammenfassende Bemerkung, die auch als solche signalisiert wird ("Zusammenfassend gesehen, stehen so die Grundsätze der Ausführung des EGR [...] gegenüber dem nationalen Recht unter dem Leitgedanken, die reale Verwirklichung der "Rechtsgemeinschaft" EG so leicht, vollständig und dauerhaft wie irgend möglich zu machen [...]", S. 206); ansonsten ist in den deutschen Texten keine weitere metakommunikative Angabe mehr zu finden.

Was hingegen im Deutschen in großem Maße vertreten ist, ist die Verbindung durch Verweise auf vorherige oder nachfolgende Themen, sei es durch bloße Angabe der entsprechenden Randnummer, der Seite oder des Abschnittes, sei es - wenn auch in geringerem Maße - durch verbale Hervorhebung.

Solche deiktischen Signale verweisen oft auf Stellen, wo für die erwähnten Begriffe (Institute, Maßnahmen usw.) eine Definition und eine Erklärung zu finden ist, so daß der Leser in die Lage versetzt wird, schnell und praktisch (auch dank der Randnummer- bzw. Randziffermarkierung) auf Grundlagenwissen zurückzugreifen, wie im folgenden Beispiel aus Oppermann (S. 200), wobei unter der zitierten Randziffer die Rede von "perfekten" Richtlinien ist:

Vergleichbares gilt, wenn "perfekte" Richtlinien oder an Mitgliedstaaten gerichtete Entscheidungen von den MS nicht fristgemäß durchgeführt werden (Näher oben Rz: 466).

Verweise werden darüber hinaus auch verbal expliziter signalisiert, wie in den Beispielen

Wie schon anderwärts bei den Fortentwicklungs- und Auflösungsmöglichkeiten der EGen festgestellt wurde (oben Rz. 187ff.), widerstrebt zwar der einmal erreichte, definitiv gemeinte "Gemeinschaftsbestand" in starkem Maße jedem Rückschritt. Dennoch bleiben die Gemein- 
schaften und ihr Recht bis auf weiteres fragilere Gebilde als ihre MS und in ihrer Entwicklung von deren politischem Willen und dem "europäischen Erfolg" abhängig (Oppermann, S. 198).

Allerdings waren, wie oben gezeigt, damit nicht alle Fragen gelöst (Bleckmann, S. 316).

Alles in allem scheint die Orientierung des Lesers in den deutschen Texten aber eher durch die starke Gliederung und Strukturiertheit der Kapitel gesteuert zu werden als durch (verbalisierte) textuelle Deixis und metatextuelle Hinweise durch den Autor; in der Ökonomie dieser Texte würde sich der Einsatz solcher Mittel vermutlich auch nicht als so produktiv erweisen, da Themenwechsel und inhaltliche Einheiten, wie vorher gezeigt, sowohl struktural als auch graphisch hervorgehoben werden.

Dasselbe gilt nicht für das italienische Korpus, das durch eine höhere Frequenz auktorialer Hinweise hinsichtlich der Progression der Behandlung gekennzeichnet ist.

Bei vier von fünf Lehrtexten erwähnt der Autor nämlich in den einleitenden Sätzen oder im ersten Abschnitt des Kapitels das zu behandelnde Hauptthema, wobei er auch erklärt, aus welcher Perspektive die Darstellung erfolgen wird. Bei dieser Themaeinleitung wird auf das Thema des vorherigen Kapitels Bezug genommen und dadurch die Kontinuität zwischen den Kapiteln gewährleistet. Eine solche Verknüpfung geht bei einem der Lehrbücher (Pocar) so weit, daß am Ende jedes Abschnitts das Thema des folgenden Abschnittes im voraus thematisiert wird (siebenmal insgesamt). Diese Tatsache ist um so bedeutender, wenn man bedenkt, daß gerade Pocar nur eine Gliederungsebene aufweist und daß bei ihm keine typographischen Hervorhebungen eingesetzt werden.

Auch in Ballarino zeigt der Autor seine Präsenz im Text sehr deutlich, indem er am Anfang des Kapitels das Problem nach einzelnen Aspekten gliedert (und somit auch die thematische Gliederung des Kapitels deutlich macht), indem er die besondere ausgewählte Perspektive erwähnt und indem er die Hauptthemen des Kapitels einigemal explizit wieder aufgreift. Themaeinleitung, Verknüpfung mit vorher behandelten Themen und Themaantizipierung sind auch in Ferrari Bravo zu finden; hier werden außerdem Themaänderungen und Digressionen signalisiert. Ähnliche Explizitheit ist auch bei Zanghì festzustellen (Themaeinleitung am Kapitelanfang und bei zwei Kapitelabschnitten); die wenigsten metatextuellen Orientierungsmittel verwendet Lauria, wo nur in einem Abschnitt Bezug auf das vorherige und auf das aktuelle Thema genommen wird.

Auch bei intratextuellen Verweisen, die sprachlich ausgedrückt werden und die auf Stellen in vorhergehenden oder nachfolgenden Abschnitten verweisen, scheint sich bei den italienischen Autoren eine etwas höhere Frequenz und damit eine größere Kohäsion zu bestätigen $(10,7,5,2,0$ im Vergleich zu 2, 1, 1,0), während die deutschen Autoren mehr Verweise verwenden, die aus einfachen Formen wie "siehe oben/unten" und aus Seiten- bzw. Zifferverweisen bestehen.

Insgesamt gesehen, scheinen die italienischen Autoren eine stärkere Tendenz zu haben, den Leser mit Hilfe verbaler Mittel (insbesondere metatextueller Hinweise) zu orientieren, was die geringere Anwendung von strukturellen oder graphischen 
Verfahren ausgleicht; hingegen sind die deutschen Texte visueller organisiert, so daß sie auf die sprachliche Signalisierung verzichten.

\subsection{Komplexität auflösen und Komplexität darstellen: Erklären und Präzisieren}

Unter den metakommunikativen Verfahren, die eingesetzt werden können, um wie Baumann erklärt - "die Verständlichkeit sprachlich-kommunikativen Handelns zu sichern" (Baumann 1995: 121) schienen solche Verfahren zur kontextspezifischen Rezeption des Fachtextes wie Erklärungen und Präzisierungen für juristische Lehrbücher besonders relevant zu sein, da es hier darum geht, komplexe Sachverhalte so zu vermitteln, daß sie in ihrer ganzen Komplexität völlig klar verstanden werden. ${ }^{16}$

In der Korpusanalyse wurde daher nach Elementen gesucht, die explizit eine Erklärung oder eine Präzisierung einleiten: Es sind dies Junktoren wie das heißt, nämlich, und zwar, die jeweils eine "nachgeschobene Erklärung" oder eine "erklärende Präzisierung eines vorhergehenden Ausdrucks oder Satzes" einleiten und "nachträgliche Erläuterungen, Präzisierungen" zum Ausdruck bringen (Engel 1988: 742-747). Hinzu kommen Ausdrücke wie mit anderen Worten, anders ausgedrückt und ähnliche, die eine Paraphrase und letzten Endes eine Erklärung einleiten. Für das Italienische werden solche Funktionen von Junktoren wie cioè, ovvero, ossia und Ausdrücke wie o meglio, in altri termini, in altre parole und ähnliche ausgeübt. Beispiele dafür sind:

Entscheidend für eine "europäisch" inspirierte Lösung der Vorrangfrage ist die Anerkennung des Grundgedankens, daß das Europäische Gemeinschaftsrecht eine eigenständige Rechtsordnung mit einer autonomen Gemeinschaftsgewalt darstellt. Sie ist von derjenigen der Mitgliedstaaten unabhängig, d.h.h das EGR entsteht aus sich selbst heraus gemäß seinen besonderen Grundregeln (Oppermann, S. 196).

Kelsen steht vor einem zweiten Problem bei der Frage, welche Befugnisse das Völkerrecht den Staaten denn eingentlich einräumt. Um die Souveränität, d.h. die Fülle der Hoheitsgewalt kann es sich dabei wohl kaum handeln [...] (Bleckmann, S. 331).

Begründet hat [der $\mathrm{EuGH}$ ] das damit, daß bestimmte Normen so formuliert sind, daß sie:

a) rechtlich vollkommen sind, d.h. ohne jede weitere Konkretisierung anwendbar sind; [...] (Schweitzer/Hummer, S. 213).

[Art. 24 GG] eröffne deshalb nicht den Weg, die Grundstruktur der Verfassung, auf der ihre Identität beruhe, ohne Verfassungsänderung, nämlich durch die Gesetzgebung der zwischenstaatlichen Einrichtung, zu ändern (Bleckmann, S. 317 ).

Indem Art. 189 II Konstitutionsnorm der Gemeinschaft geworden sei, habe die Bundesrepublik verfassungskräftig, und zwar mit Art. 24 I GG als "Integrationshebel", die Existenz, die Geltung und die Wirksamkeit von Gemeinschaftsnormen in ihrem Staatsgebiet neben der nationalen Rechtsmasse anerkannt [...] (Bleckmann, S. 301).

16 Zur Rolle der Erklärung als metakommunikativer Strategie bei didaktischen Texten siehe auch Gläser (1995: 90). 
[...] successivamente si è posto il quesito se vi sia luogo per distinguere l'effetto diretto, cioè la possibilità aperta ai singoli di invocare una norma di diritto comunitario diversa dai regolamenti avanti ad un organo giudiziale nazionale, dall' applicabilità diretta [...]. (Ballarino, S. 231)

È degno di nota comunque il limite che la Corte costituzionale pone a tale suo atteggiamento: "Il sindacato di costituzionalità può esercitarsi solo sulle statuizioni della legge interna, denunciate avanti ad essa in quanto dirette a pregiudicare la perdurante osservanza del Trattato di Roma". In altre parole, il metodo della "disapplicazione" da parte del giudice comune non è sufficiente [...] per le leggi interne che fanno ostruzionismo alla costruzione comunitaria. (Ballarino, S. 253)

Nun zeigt die Textanalyse, daß solche expliziten Mittel nur in begrenztem Maße verwendet werden, besonders in den deutschen Texten, wo sich die Okkurrenz solcher Junktoren innerhalb einer Skala von 2 bis 8 bewegt $(2,3,4,6,8$, bei einem Textumfang von 10 bis 25 Seiten), während dieses Verfahren in den italienischen Texten in einem etwas höheren Maße (2, 2, 5, 13, 15, bei einem Textumfang von 12 bis 32 Seiten) eingesetzt wird. Dieses Bild verändert sich leicht, wenn man dazu auch anders signalisierte Erklärungen zählt wie z.B. solche, die durch graphische Zeichen wie Klammer eingeleitet werden. So werden in den deutschen Texten jeweils 3, 6, 12, 17, 18 Erklärungen sprachlich oder nichtsprachlich signalisiert und bei den italienischen Texten jeweils $2,8,9,18,42$. Beispiele dafür sind:

In den kleineren MS (Belgien, Dänemark, Griechenland, Irland, Luxemburg, Niederlande) ist der Vorrang des EGR vor dem nationalen Recht bisher nicht ernsthaft in Frage gestellt worden (Oppermann, S. 198).

Dem Gemeinschaftsrecht kommt vor nationalem Recht Vorrang zu. Die dafür gegebenen Begründungen unterscheiden sich allerdings nicht nur in den einzelnen Argumenten, sondern auch im Grundsätzlichen, und auch die Art des Vorrangs (Geltungs- oder Anwendungsvorrang) wird unterschiedlich gesehen (Streinz, S. 54).

Tuttavia, il fenomeno comunitario c'era e a un certo punto cominciò ad infittirsi la presenza delle norme derivate (regolamenti, direttive, decisioni) ed iniziò lo sviluppo della giurisprudenza della Corte di Giustizia [...] (Ferrari Bravo, S. 152).

Während Erklärungen nicht so sehr signalisiert werden, wie man von Lehrbüchern erwarten könnte - was auch den relativ hohen Fachlichkeitsgrad des analysierten Korpus beweist -, so werden Präzisierungen sehr oft im Korpus graphisch repräsentiert und somit hervorgehoben, selbst wenn sie formal nicht zur Hauptdarstellung gehören und sozusagen eine zweite, vertiefende Ebene der Informationsvermittlung darstellen. Präzisierungen werden dabei eingesetzt, um eine vorhergehende Information zu ergänzen oder begrenzen, um historisches, politisches oder fachliches Hintergrundwissen zu liefern, Beispiele anzuführen und die Meinung des Autors widerzuspiegeln. So findet man z.B. im deutschen Korpus:

Die europarechtlichen Lösungen können - insbesondere was ihr Ergebnis des Vorrangs des Gemeinschaftsrechts anbelangt - als die herrschende Lehre bezeichnet werden [...] (Schweitzer) Hummer, S. 215). 
Zum Teil mußten erst erhebliche Schwierigkeiten überwunden werden, die - wie die Überprüfung von Gemeinschaftsrecht an nationalem Verfassungsrecht - auch heute noch nicht vollständig gelöst sind (Beutler/Bieber, S. 98).

1979 hat das BVerfG den "Solange I-Beschluß" durch den sog. "Vielleicht-Beschluß" - wohl als Reaktion auf die heftige Kritik von seiten der. Wissenschaft - ein wenig relativiert, indem es ausführte [...] (Schweitzer/Hummer, S. 218).

Ähnliches ist auch im Italienischen dokumentiert:

La Corte nella sentenza afferma: "[...] La norma significa che, quando ricorrono certi presupposti, è possibile stipulare trattati con cui si assumono limitazioni della sovranità ed è consentito di darvi esecuzione con legge ordinaria" (e questo è corretto) (Ferrari Bravo, S. 157).

La relazione diritto comunitario/diritto interno (dirò subito che questa relazione non può essere esposta in termini di rapporto "diritto internazionale/diritto interno quanto alle materie regolate dai trattati") deve quindi essere configurata in termini differenti (Ballarino, S. 229).

Soltanto le direttive e le decisioni - in quanto, naturalmente, si tratti di direttive non suscettibili di applicazione immediata e di decisioni che si rivolgono a Stati membri e non ad individui abbisognano dunque di misure di esecuzione negli Stati membri [...] (Pocar, S. 316).

Die Häufigkeit solcher Präzisierungen, Ergänzungen und Einschränkungen scheint höher als diejenige der Erklärungen zu sein, bedingt vermutlich auch durch die Komplexität des Stoffes; bei dem Vergleich zeigt sich eine größere Ausgewogenheit in der Verwendung solcher Verfahren in den deutschen Texten (mit 21, 19, 19, 16, 15 Okkurrenzen), während es bei den italienischen Autoren von einem Extrem ins andere geht $(49,19,13,3,0)$.

Die Analyse dieser beiden Strategien zeigt den schon relativ hohen Fachlichkeitsgrad solcher Lehrbücher: der Student steht fast am Ende seiner 'Sozialisierung' als Jurist und muß in der Lage sein, sich mit komplexen Sachverhalten, die auch in komplexer Weise präsentiert werden, zurechtzufinden; ihm müssen die wichtigsten juristischen Begriffe schon vertraut sein und er muß den vielen 'Sprüngen' zwischen Haupt- und Nebeninformation, wie sie z.B. bei Präzisierungen realisiert werden, folgen können.

\subsection{Die Signalisierung der kausalen Beziehungen als Rezeptionssteuerung}

Unter den Mitteln, die einem Autor für die Steuerung der Textrezeption zur Verfügung stehen, spielen Kohäsionsmittel eine wichtige Rolle. Kohäsionsmittel dienen dazu, semantisch-logische Relationen explizit an der Textoberfläche zu zeigen; sie können damit dem Leser das Textverstehen erleichtern, indem sie gewisse Signale zur Interpretation der Beziehungen zwischen den einzelnen Texteinheiten liefern. ${ }^{17}$ Bei Lehrtexten scheint deswegen die Verwendung von Kohäsionsmitteln von besonderer Relevanz zu sein, weil sie dem Autor sozusagen die 'Kontrolle' über die potentielle

17 Vgl. dazu de Beaugrande / Dressler (1981), Gislimberti (1988). 
Rezeption seitens des Lesers garantiert und damit auch eine Garantie über die Qualität der gelieferten Information (die vom Studenten gelernt und aufgenommen werden soll) gibt.

Das ausgewählte Thema - das Verhältnis von Gemeinschaftsrecht und nationalem Recht - gilt seit Beginn der Gemeinschaft als eines der kontroversesten; der Autor behandelt dieses Thema, indem er die Argumente verschiedener Instanzen (Europäischer Gerichtshof, nationale Verfassungsgerichte, die in der Rechtslehre vertretenen Positionen) darstellt, kommentiert, unterstützt oder widerlegt. Die ausgewählten Textabschnitte sind also zwar expositorisch, sie besitzen aber wegen des besonderen Themas auch einen argumentativen Charakter, indem sie externe Argumentationen wiedergeben und erklären und auktoriale Positionen ausdrücken.

So wurde bei der vorliegenden Untersuchung besonderes Augenmerk auf die Signalisierung von kausalen Relationen (wobei auch konsekutive Beziehungen einbezogen wurden) an der Textoberfläche gelegt, d.h. auf das Vorhandensein von kohäsiven Elementen, die eine Ursache-Wirkung-Beziehung oder eine Grund-FolgeBeziehung kennzeichnen. Hier wurde in erster Linie nach den "Nexusadverbien" gesucht, die unter der Argumentationsadverbien die Aufgabe haben,

eine Feststellung so in den Argumentationsgang einzubinden, daß sie zu dem voraufgehenden Kontext oder zur Situation in Beziehung gesetzt wird. Diese Adverbien bringen eine Begründungsbeziehung zwischen zwei Prädikationen zum Ausdruck. Sie haben daher das primäre Merkmal (BEGRÜNDUNG). Mit Nexus-Adverbien kann der Sprecher zum Beispiel seinem Gesprächsparnter mitteilen, daß der zuvor festgestellte Sachverhalt als Stütze seiner Argumentation anzusehen ist. Umgekehrt kann er aber auch anzeigen, daß ein vielleicht naheliegendes Argument von einem Sachverhalt nicht gestützt wird (Weinrich 1993: 600).

Dazu gehören in der deutschen Sprache die Adverbien daher, darum, denn, deshalb, deswegen, nämlich und Adverbien wie also, folglich, demnach, mithin, infolgedessen, demzufolge, insofern, somit, die "für einen Argumentationsschritt, der eine längere Argumentationskette mit einer Folgerung abschließt" (Weinrich 1993: 603) gebraucht werden. Neben solchen "textuellen Operatoren" (Gislimberti 1988: 75) wurden auch "Satzoperatoren" wie die Konjunktionen weil und da berücksichtigt. Im Italienischen stehen ähnliche Operatoren zur Verfügung (quindi, dunque, perciò, di conseguenza, infatti; perché, poiché, dato che, dal momento che, tanto più che) ${ }^{18}$

Bei dem Vergleich zwischen den beiden Korpora zeigt sich die Tendenz, kausale Verhältnisse im Italienischen in höherem Maße als im Deutschen explizit zu signalisieren; italienische Autoren scheinen somit einen etwas höheren Gebrauch von argumentativen Mitteln als deutsche Autoren zu machen, um dem Leser den komplexen Sachverhalt zu vermitteln (siehe Tabelle 1).

Eine solche Annahme sollte natürlich durch die detaillierte Analyse aller Kohäsionsmittel bestätigt werden, die für die Argumentation und sonderlich für die 
Signalisierung kausaler Verhältnisse verwendet werden;19 allerdings scheinen diese ersten und begrenzten Beobachtungen die Ergebnisse kontrastiver Untersuchungen zur Kausalität zu bestätigen, die eine stärkere Tendenz des Italienischen zur Kohäsion sowie eine stärkere Tendenz des Deutschen zur Kohärenz (wobei kausale Beziehungen nicht explizit an der Textoberfläche signalisiert werden, sondern implizit sind) belegen.

Es handelt sich dabei um Analysen zu den Textsorten "Wirtschaftszeitungskommentar" (Gislimberti 1988) und "richterliche Entscheidungsbegründung" (als Teil des Gerichtsurteils) (Wiesmann 1994); bei der Textsorte 'Zeitungskommentar' wird stärkere Signalisierung von Kausalität und folglich stärkere Empfängergerichtetheit "mit einer tendenziell persuasiven Textfunktion verbunden, schwächere Signalisierung und folglich schwächere Empfängergerichtetheit dagegen mit einer tendenziell informativen Textfunktion" (Wiesmann 1994: 6). Bei der Textsorte "Entscheidungsbegründung" wird parallel dazu die stärkere Tendenz zur Kohäsion im Italienischen und die schwächere Tendenz zur Kohäsion im Deutschen als Ausdruck unterschiedlicher Senderintention gewertet und in Zusammenhang "mit einer Textfunktion gesehen, die sich zwischen den Polen informativ und persuasiv bewegt" (Wiesmann 1994: 6).

Wenn bei der Textsorte "Lehrbuch" eine höhere Verwendung von kausalen Konnektoren im Italienischen zu finden ist, so scheint dies die eben genannte Tendenz italienischer Autoren zu bestätigen, einen Sachverhalt eher argumentativ als deskriptiv darzustellen; andererseits übt eine solche Signalisierung bei den italienischen Lehrbüchern eine besondere Orientierungsfunktion aus, die bei den deutschen nicht so notwendig zu sein scheint. Die italienischen Texte - wie vorher gesehen - verwenden nicht die ganze Palette an Gliederungs- und Orientierungsmitteln, die bei den deutschen eingesetzt werden (starke Strukturierung, graphische Hervorhebung); Sätze sind in der Regel im italienischen Korpus länger, was auch die Rezeption nicht erleichtert. Dadurch scheint der größere Einsatz sprachlicher Elemente, darunter auch kausaler Konnektoren, eine fast notwendige Maßnahme zu sein, um die Textrezeption zu steuern.

Solche Verhältnisse können durch Präpositionen (wie z.B. wegen, aufgrund, durch, infolge, mittels, zufolge bzw. per, di, da, a causa di, per causa di, per motivi usw.) lexikalische Mittel (Grund, Ursache bzw. causa, motivo, ragione; Anlaß geben zu, Anlaß sein für, zur Folge haben, auslösen, begründen, mit sich bringen, bringen zu, ergeben, erzeugen, führen zu, veranlassen, verursachen bzw. avere come conseguenza, avere come risultato, conseguire, causare, comportare, condurre a, indurre a, motivare, usw.) und implizite Formen (Partizipialkonstruktion im Deutschen, Partizipialkonstruktion, Gerundium und Infinitiv im Italienischen) ausgedrückt werden. Vgl. dazu Gislimberti (1988: 73-74). 


\section{Abschließende Bemerkungen}

Bei der hier dargestellen Analyse wurden intuitiv ausgewählte Vergleichsparameter auf ein Korpus von juristischen Lehrbüchern angewendet, die gewisse Unterschiede aufzuweisen schienen. Es sollte nachgeprüft werden, inwieweit sich solche Vergleichsgrößen für die kontrastive Untersuchung von didaktischen Texten eignen; konkret konnten einige Tendenzen gezeigt werden, die auf eine Differenzierung zwischen deutschen und italienischen Texten hindeuten und die durch größere Untersuchungen zu bestätigen sind.

Bei dem Vergleich haben Vorwörter als erster 'Begegnungsort' zwischen Autor und Leser eine stärkere Adressatenorientierung bei den deutschen Texten gezeigt; Elemente wie Gliederung und typographische Signale haben eine größere 'visuelle' Tendenz im Deutschen gezeigt. Die Analyse der deiktischen Ausdrücke, insbesondere der metakommunikativen Äußerungen des Autors über die Progression seiner eigenen Darstellung hat hingegen eine stärkere Tendenz des Italienischen zur Versprachlichung und zur expliziten Signalisierung ergeben. Die relativ geringe Verwendung von (expliziten) Erklärungen hat vor allem den hohen Fachlichkeitsgrad der analysierten Lehrbücher gezeigt, der auch durch die besondere Rolle von Präzisierungen als Vertiefungsebene bestätigt wird. Schließlich wurde durch die Analyse von kohäsiven Elementen (Nexus-Adverbien und Konjunktionen), die ein kausales Verhältnis signalisieren, die schon bei anderen Arbeiten anerkannte Tendenz im Italienischen bestätigt, Kausalität expliziter als im Deutschen zu signalisieren und damit den Leser in der Textrezeption in größerem Maße zu steuern.

Die ausgewählten Parameter haben sich insofern für eine solche kontrastive Untersuchung als geeignet erwiesen; deren Anwendung hat auch gezeigt, in welche Richtung die Analyse weitergehen könnte, um einerseits die hervorgehobenen unterschiedlichen Tendenzen in den zwei Sprachen zu bestätigen und um andererseits Ähnlichkeiten im Rahmen einer allgemeineren Beschreibung des juristischen Diskurses einbetten zu können. Dabei ist insbesondere auf die Bereiche der Argumentation und der Kohäsion gedacht: eine umfangreichere Analyse der argumentativen Mittel, die Meinung und Haltung des Autors gegenüber dem behandelten Thema explizit machen, ${ }^{20}$ wird es ermöglichen, die angenommene Tendenz des Italienischen zur größeren Explizitheit zu bestätigen oder zurückzuweisen; die Erweiterung der Untersuchung auf kohäsive Mittel wie z.B. Rekurrenz, Paraphrase und Elemente, welche die (temporale, lokale oder logische) Artikulierung des Textes ${ }^{21}$ unterstreichen, wird ein angemesseneres Bild der Strategien geben, die ein Fachmann in der Vermittlung von komplexen Sachverhalten an einen 'fortge-

Dies sind z.B. Geltungsadverbien, Adverbien zum Ausdruck von Konzessivität und Adversativität und von Bedingung und Folge, lexikalische Operatoren.

21 Man denke an kohäsive Ketten wie zunächst...dann...inzwischen...schließlich, auf der einen Seite...auf der anderen Seite u.ä. Vgl. dazu auch Sabatini (1990: 689). 
schrittenen' Laien einsetzt und mit denen er zu dessen wissenschaftlicher Sozialisierung beiträgt. Der Vergleich mit anderen juristischen Lehrbüchern einerseits und mit Lehrbüchern anderer Fächer andererseits könnte schließlich die Besonderheiten des juristischen Diskurses in vertikaler und horizontaler Hinsicht deutlicher machen.

\begin{tabular}{|l|c|c|c|c|c|c|c|c|c|c|c|c|}
\hline & B & F & L & P & Z & & BL. & BEU & OPP & SCH & ST & \\
\hline Satzzahl & 195 & 298 & 130 & 146 & 122 & & 212 & 201 & 130 & 109 & 146 & \\
\hline $\begin{array}{l}\text { Textuelle Opera- } \\
\text { toren }\end{array}$ & & & & & & & & & & & & \\
\hline quindi & 8 & 17 & 11 & 10 & 6 & daher & - & 10 & 3 & 3 & 4 & \\
\hline pertanto & 3 & 1 & 2 & 2 & - & deshalb & 8 & 10 & - & - & 3 & \\
\hline perciò & 2 & 3 & - & 3 & - & darum & - & - & - & 2 & - & \\
\hline dunque & 1 & 2 & 3 & 7 & - & also & 17 & 3 & 3 & 2 & 1 & \\
\hline infatti & 8 & 4 & 5 & 12 & 5 & nämlich & 6 & & - & 2 & 2 & \\
\hline di conseguenza & - & 1 & 4 & - & 2 & folglich & 2 & - & - & 1 & - & \\
\hline & & & & & & deswegen & 1 & & - & - & 3 & \\
\hline & & & & & & somit & - & - & 1 & 2 & - & \\
\hline & & & & & & denn & 9 & 5 & & & 3 & \\
\hline $\begin{array}{l}\text { Gesamt (absolute } \\
\text { Zahlen) }\end{array}$ & 22 & 27 & 25 & 34 & 11 & & 43 & 21 & 7 & 12 & 16 & \\
\hline Verhältnis & $1 / 8$ & $1 / 12$ & $1 / 5$ & $1 / 4$ & $1 / 11$ & & $1 / 5$ & $1 / 10$ & $1 / 18$ & $1 / 9$ & $1 / 9$ & \\
\hline Satz-Operatoren & & & & & & & & & & & & \\
\hline $\begin{array}{l}\text { perché, poiché, } \\
\text { dato che, dal mo- } \\
\text { mento che, tanto } \\
\text { più che }\end{array}$ & 8 & 11 & 14 & 9 & 4 & weil, da, & 12 & 11 & 2 & 4 & 11 & \\
\hline Gesamtsumme & 30 & 38 & 39 & 43 & 15 & & 55 & 32 & 9 & 16 & 27 & \\
\hline Verhältnis & $1 / 6$ & $1 / 8$ & $1 / 3$ & $1 / 3$ & $1 / 8$ & & $1 / 4$ & $1 / 6$ & $1 / 14$ & $1 / 7$ & $1 / 5$ & \\
\hline
\end{tabular}

Tabelle 1: Distribution der kausalen Konnektoren

\section{Legenda:}

Italienische Lehrbücher: $\mathrm{B}=$ Ballarino; $\mathrm{F}=$ Ferrari Bravo; $\mathrm{L}=$ Lauria; $\mathrm{P}=$ Pocar; $\mathrm{Z}=$ Zanghì.

Deutsche Lehrbücher: $\mathrm{BL}=$ Bleckmann; BEU= Beutler/Bieber; OPP= Oppermann; $\mathrm{SCH}=$

Schweitzer/Hummer; ST= Streinz.

Verhältnis: die Gesamtzahl der Konnektoren steht hier im Verhältnis zur Gesamtzahl der Sätze (durchschnittliche Frequenz der Konnektoren). 


\section{Zusammenfassung}

Im Zentrum des vorliegenden Beitrags steht die explorative Analyse von deutschen und italienischen juristischen universitären Lehrbüchern zum Europarecht. Um herauszufinden, wie sich der Dialog zwischen Fachmann und Laien bei solchen Texten profiliert und wie sich die Didaktisierung der Inhalte in den zwei Sprachen realisiert, wurden paratextuelle und textinterne Vergleichsparameter wie Vorwörter, Gliederung, graphische und typographische Gestaltung, deiktische und metakommunikative Ausdrücke, Erklärungen und Präzisierungen, und kausale Konnektoren untersucht. Aus der vergleichenden Analyse geht eine größere Aufmerksamkeit der deutschen Autoren gegenüber dem Leser hervor, was Hilfsmittel und strukturelle, graphische und typographische Orientierungsmittel anbelangt; italienische Autoren scheinen hingegen mehr Gebrauch von verbalen Mitteln (Metakommunikation, Explizierung von Kausalität) zu machen, um den Leser im Text zu orientieren. Diese Ergebnisse, die keine Repräsentivität beanspruchen sondern exemplarischen Charakter haben, sollen in einer zweiten Phase der Untersuchung, die weitere Vergleichsparameter einbezieht, bestätigt werden.

\section{Bibliographische Hinweise}

\section{Quellenverzeichnis}

\section{Deutsche Lehrbiicher:}

Beutler, B. / Bieber, R. / Pipkorn, J. / Streil, J. (1993): Die Europäische Union: Rechtsordnung und Politik, Baden Baden, 4. Auflage, 94-112.

Bleckmann, A. (1990): Das Recht der Europäischen Gemeischaft, 5. Auflage, 297-333. Opperman, T. (1991): Europarecht. Ein Studienbuch, München, 194-206.

Schweitzer, M. / Hummer, W. (1993): Europarecht, 4. Auflage, 212-224

Streinz, R. (1995): Europarecht, Heidelberg, 2. Auflage, 51-66.

Italienische Lehrbücher:

Ballarino, T. (1993): Lineamenti di diritto comunitario, Padova, 225-267.

Ferrari Bravo, L. / Moavero Milanesi, E. (1995): Lezioni di diritto comunitario, Napoli, 151-170.

Lauria, F. (1992): Manuale di diritto delle Comunità Europee, Torino, 3. Auflage, 173-190.

Pocar, F. (1991): Diritto delle Comunità Europee, Milano, 4. Auflage, 309-345.

Zanghì, C. (1995): Istituzioni di diritto dell'Unione Europea, Torino, 327-240.

\section{Literatur}

Arntz, R. (Hrsg.) (1995): La traduzione. Nuovi approcci tra teoria e pratica. Napoli.

Baumann, K.-D. (1995): "Die Verständlichkeit von Fachtexten. Ein komplexer Untersuchungsansatz". In: Fachsprache 3-4/1995. 116-126.

Baumann, K.-D. / Kalverkämper, H. (Hrsg.) (1992), Kontrastive Fachsprachenforschung, Tübingen. 
Beaugrande de R.-A. / Dressler, W.U. (1981): Einführung in die Textlinguistik. Tübingen.

Bertocchi, D. et alii (1988): "Analisi di manuali scientifici ed ipotesi di leggibilità". GISCEL Lombardia. In: Guerriero, A. R. (Hrsg.): L'educazione linguistica e $i$ linguaggi delle scienze. Firenze. 239-265.

Busch-Lauer, I. (1994): "Kontrastive Fachtextanalyse am Beispiel englischer und deutscher Zeitschriftenartikel der Medizin". In: Spillner, B. (Hrsg.): Fachkommunikation. Kongreßbeiträge zur 24. Jahrestagung der Gesellschaft für Angewandte Linguistik. Frankfurt a.M. 71-72.

Cavagnoli, S. / Veronesi, D. (1996): "Fachsprachliche Lesekompetenz. Italienisch für Juristen und Wirtschaftler". In: Fachsprache 3-4/1996. 150-159.

Clyne (1993): "Pragmatik, Textstruktur und kulturelle Werte. Eine interkulturelle Perspektive". In: Schröder, H. (Hrsg.): Fachtextpragmatik. Tübingen. 3-18.

Conte, M.-E. (1984): "Deixis am Phantasma. Una forma di riferimento nei testi". In: Coveri, L. (Hrsg.): Linguistica testuale. atti del XV congresso internazionale di studi. Roma. 187-205.

Cortelazzo, M. (1994): Lingue speciali. La dimensione verticale. Padova.

Desideri, P. (1991): "Il testo argomentativo: processi e strumenti di analisi". In: Desideri, P. (Hrsg.): La centralità del testo nelle pratiche didattiche. Firenze. 121-143.

Ehlich, K. (1984): "Deictic Expressions and the Connexity of Text". In: Conte, M-E. / Petöfi, J.S. /Sözer, E. (Hrsg.): Text and Discourse Connectedness. Proceedings of the Conference on Connexity and Coherence, Urbino, July 16-21, 1984. Amsterdam. 33-52.

Engel, U. (1988): Deutsche Grammatik. Heidelberg.

Fiedler, S. (1994): "Linguistische Fachtexte im Englischen, Deutschen und Esperanto (ein interkultureller Vergleich". In: Spillner (Hrsg.) Fachkommunikation. Kongreßbeiträge zur 24. Jahrestagung der Gesellschaft für Angewandte Linguistik. Frankfurt a.M. 68-71.

Genette, G. (1987): Seuils, Paris.

Gislimberti, S. (1988): Coesione testuale. Un'analisi contrastiva (tedesco - italiano) di commenti della stampa quotidiana. Wilhelmsfeld.

Gläser, R. (1990): Fachtextsorten im Englischen. Tübingen.

Gläser, R. (1992): "Methodische Konzepte für das Tertium comparationis in der Fachsprachenforschung". In: Baumann / Kalverkämper (Hrsg.): Kontrastive Fachsprachenforschung, Tübingen. 78-92.

Gläser, R. (1994): "Die Textsorte "Sachbuch" im Englischen und Deutschen". In: Spillner, B. (Hrsg.): Fachkommunikation. Kongreßbeiträge zur 24. Jahrestagung der Gesellschaft für Angewandte Linguistik. Frankfurt a.M. 75-78.

Gläser, R. (1995): Linguistic Features and Genre Profiles of Scientific English. Frankfurt a.M.

Gnutzmann, G. (1992): "Kontrastive Fachtextlinguistik als Projektaufgabe. Theoretische Fragen und praktische Antworten". In Baumann / Kalverkämper (Hrsg.): Kontrastive Fachsprachenforschung, Tübingen. 266-275.

Göpferich, S. (1995). "A Pragmatic Classification of LSP Texts in Science and Technology". In Target 7:2/1995. 305-326. 
Hoffmann, L. (1984): Kommunikationsmittel Fachsprache. Eine Einführung. Berlin.

Kalverkämper, H. (1993): "Das fachliche Bild. Zeichenprozesse in der Darstellung wissenschaftlicher Ergebnisse". In: Schröder, H. (Hrsg.): Fachtextpragmatik. Tübingen. 215-238.

Kühn, P. (1992), Bausteine Fachdeutsch für Wissenschaftler: Jura. Heidelberg.

Lewandowski, Th. (1990): Linguistisches Wörterbuch. Heidelberg.

Oldenburg, A. (1995): "Methodologische Grundlangen der kontrastiven Fachtextlinguistik". In: Fachsprache 3-4/1995. 107-116.

Pöckl, W. (1995): "Nationalstile in Fachtexte? Vom Tabu- zum Modethema". In: Fachsprache 3-4/1995. 98-107.

Sabatini, F. (1990): "Analisi del linguaggio giuridico. Il testo normativo in una tipologia generale dei testi". In: D'Antonio, M. (Hrsg.): Corso di studi superiori legislativi 1988 -89. Padova. 675-724.

Sachtleber, S. (1993): "Textstile in der Wissenschaftssprache". In: Schröder, H. (Hrsg.): Fachtextpragmatik. Tübingen. 61-79.

Scarpelli, U. / Di Lucia, P. (Hrsg.) (1994): Il linguaggio del diritto. Milano.

Vanelli, L. / Renzi L. (1995): "La deissi". In: Renzi L./ Salvi, G. / Cardinaletti, A. (Hrsg.): Grande grammatica italiana di consultazione. Vol. III: Tipi di frase, deissi, formazione delle parole. Bologna. 261-376.

Veronesi, D. (1997): "Analisi testuale contrastiva e applicazioni didattiche: testi giuridici italiani e tedeschi a confronto". In: Akte der Tagung Parallela VII: Italiano $e$ Tedesco in contatto e a confronto / Italienisch und Deutsch im Kontakt und im Vergleich, Innsbruck 17-19.10.1996 (erscheint demnächst).

Weinrich, H. (1993): Textgrammatik der deutschen Sprache. Mannheim.

Widdowson, H. G. (1979): Explorations in applied linguistics. Oxford.

Wiesmann, E. (1994): Kohärenz und Kohäsion. Eine Untersuchung zur Kausalität in den Entscheidungsbegründungen italienischer und deutscher Gerichtsurteile. Bologna.

Ylönen, S. (1993): "Stilwandel in wissenschaftlichen Artikeln der Medizin". In: Schröder, (Hrsg.): Fachtextpragmatik. Tübingen. 81-98.

Povzetek

PRIMERJAVA PRAVNIH BESEDIL

NEKAJ PRIMEROV ZA JEZIKOVNI PAR NEMŠČINA-ITALIJANŠČINA

Avtorica v prispevku govori o primerjalni analizi nemških in italijanskih univerzitetnih pravnih učbenikov s področja evropskega prava. Da bi ugotovila, kako poteka pri tovrstnih besedilih dialog med strokovnjakom in laikom ter kako so vsebine didaktično podane, je nemška in italijanska besedila analizirala glede na para- ter notranjebesedilne dejavnike, kot so denimo predgovor, zunanja členitev besedila, grafična in tipografska oblika, deiktični in metakomunikativni izrazi, razlage in natančnejša pojasnila ter jezikovna sredstva za ponazoritev vzročnih povezav. Rezultati analize kažejo, da nemški avtorji posvečajo več pozornosti bralcu, in sicer tako, da za njegovo lažjo orientacijo pogosteje uporabljajo pomagala (vsebinski pregled, stvarno kazalo ...), optična sredstva za ponazoritev zunanje zgradbe besedila ter grafična in tipografska sredstva. Avtorji italijanskih besedil pa bralca vodijo skozi besedilo bolj s pomočjo verbalnih sredstev (metakomunikacija, eksplikacija vzročnih relacij). Rezultati, ki niso reprezentativne, temveč eksemplarne narave, naj bi bili potrjeni v drugi fazi raziskav, ko bo treba pri analizi besedil upoštevati še nekatere druge parametre. 